\title{
BIORDERED SETS ARE BIORDERED SUBSETS OF IDEMPOTENTS OF SEMIGROUPS
}

\author{
DAVID EASDOWN
}

(Received 22 October 1982)

Communicated by T. E. Hall

\begin{abstract}
A new arrow notation is used to describe biordered sets. Biordered sets are characterized as biordered subsets of the partial algebras formed by the idempotents of semigroups. Thus it can be shown that in the free semigroup on a biordered set factored out by the equations of the biordered set there is no collapse of idempotents and no new arrows.
\end{abstract}

1980 Mathematics subject classification (Amer. Math. Soc.): 20 M 05, 20 M 10, 20 M 30, 06 A 99.

\section{Introduction}

The concept of a biordered set was introduced by Nambooripad in [2], where he proves (Theorem 1.1) that the idempotents of a semigroup form a partial algebra in a natural way which satisfies the axioms for a biordered set. The question then is whether all biordered sets arise from semigroups in this way, for then the biordered set concept will be a characterization of those partial algebras of idempotents of semigroups.

In this paper we show that all biordered sets arise as biordered subsets of the idempotents of semigroups. To do this we use a construction introduced in [1] which is the biordered set analogue of an idempotent-separating representation of semigroups.

The author does not know whether all biordered sets are the biordered sets of semigroups. A natural approach is to consider the free semigroup on the elements

(c) 1984 Australian Mathematical Society 0263-6115/84 \$A2.00+0.00 
of a given biordered set factored out by all the relations of the biordered set, and then attempt to show the biordered set of the semigroup obtained in this way coincides with the original. This breaks up into three problems: showing (i) there is no collapse of elements of the original biordered set under the factoring out by relations; (ii) no new arrows (see below) are created in the biordered set; (iii) no new idempotents are created. The main result of this paper enables (i) and (ii) to be proved; it is still not known whether (iii) occurs in general.

We begin by describing biordered sets using an arrow notation, $\rightarrow$ and $>$, to replace the more commonly used $\omega^{r}$ and $\omega^{l}$.

\section{Preliminaries}

Let $E$ be a set with a partial multiplication with domain $D_{E} \subseteq E \times E$. Define relations $\rightarrow$ and $>\subseteq E \times E$ by

$$
\begin{aligned}
& e \rightarrow f \text { if and only if }(f, e) \in D_{E} \text { and } f e=e, \\
& e>f \text { if and only if }(e, f) \in D_{E} \text { and } e f=e .
\end{aligned}
$$

Note that $e \rightarrow f$ and $e-f$ together imply $e=f$.

Suppose $E$ satisfies the following axioms.

$$
\longrightarrow \text { and }>\text { are quasi-orders and }
$$

$$
D_{E}=\rightarrow U>-U(\rightarrow U>-)^{-1} \text {, }
$$

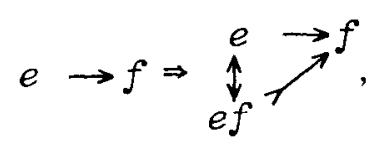

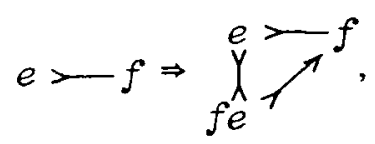

$$
\bigwedge_{f>g}^{e} \Rightarrow f e>g e
$$

$$
\stackrel{e}{f \rightarrow g} \Rightarrow e f \rightarrow e g
$$


(B31) $\quad e \rightarrow f \rightarrow g \Rightarrow(e g) f=e f$,

$(\mathrm{B} 31)^{*} \quad$ ex $f>-g \Rightarrow f(g e)=f e$,
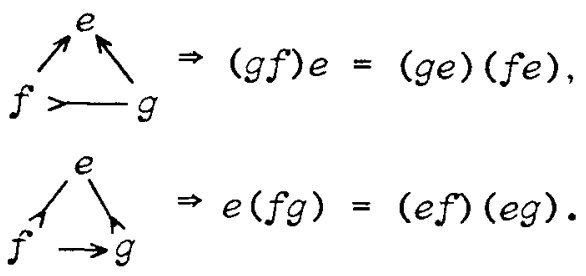

Note that successive axioms rely on previous axioms for their sense: for example in (B21), if $e \rightarrow f$ then by (B1) $(e, f) \in D_{E}$ so that $e f$ is defined; in (B31), if $e \rightarrow f \rightarrow g$ then $e \rightarrow g$ by the transitivity of $\rightarrow$, due to (B1), so by (B21) $e g \leftrightarrow e \rightarrow f$, so $e g \rightarrow f$, so that $(e g) f$ is defined. Each axiom can be made self-contained by requiring the appropriate products to be defined in $E$, but this is not necessary since $E$ will always be assumed to satisfy all these axioms.

Note that a duality exists, obtained by interchanging arrowtypes, $\rightarrow$ with $>$, and reversing the orders of products, so that (B1) is self-dual and an axiom superscripted by $*$ is the dual of the axiom without the superscript.

For $e, f \in E$ define

$$
M(e, f)=\{g \in E: g>e \text { and } g \rightarrow f\} .
$$

Define

$$
\delta(e, f)=\{h \in M(e, f): \forall g \in M(e, f) e g \rightarrow e h \text { and } g f>h f\},
$$

the sandwich set of $e$ and $f$. We call $E$ a biordered set if, in addition to the above axioms, $E$ also satisfies
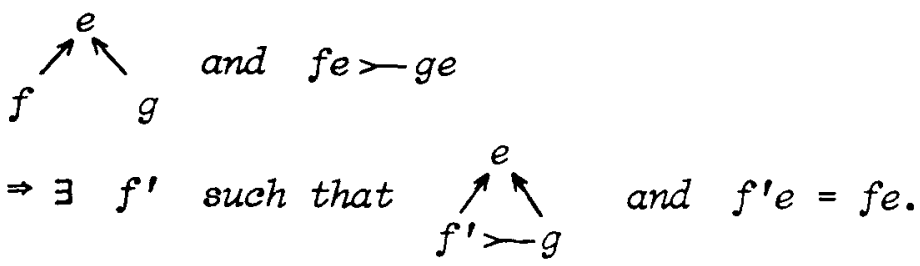

(B4)*
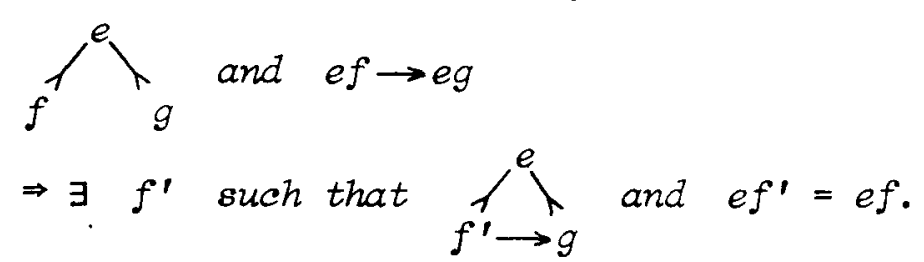

If $E$ and $E^{\prime}$ are biordered sets and $\theta: E \rightarrow E^{\prime}$ is a mapping, then $\theta$ is called a morphism if $\theta$ is a morphism of the partial algebras, that is, if $\theta$ satisfies

$$
(e, f) \in D_{E} \Rightarrow(e \theta, f \theta) \in D_{E^{\prime}} \text { and }(e f) \theta=e \theta f \theta \text {. }
$$


A subset $E^{\prime}$ of $E$ is called a biordered subset of $E$ if $E^{\prime}$ is a partial subalgebra of $E$, in the sense that if $e, f \in E^{\prime}$ and $(e, f) \in D_{E}$ then $(e, f) \in D_{E^{\prime}}$, and further $E^{\prime}$ satisfies all the biordered set axioms with respect to the restrictions of $\rightarrow$ and - to $E^{\prime}$. It is easy to see that if $E^{\prime}$ is a partial subalgebra of a biordered set $E$ then $E^{\prime}$ is a biordered subset if and only if $E^{\prime}$ satisfies (B4) and (B4)*.

The following is straightforward and proved in [2] as part of Theorem 1.1.

THEOREM 1. Let $S$ be a semigroup, and $E(S)$ the set of idempotents of $S$. Then $E(S)$ forms a biordered set by restricting the semigroup multiplication to

$$
D_{E(S)}=\{(e, f) \in S \times S: \text { ef }=e \text { or ef }=\text { for } f e=e \text { or } f e=f\} .
$$

Proposition 1 [2, Proposition (2.4)]. Let E satisfy (B1), (B21), (B21)*, (B22), $(\mathrm{B} 22)^{*},(\mathrm{~B} 31),(\mathrm{B} 31)^{*},(\mathrm{~B} 32)$ and $(\mathrm{B} 32)^{*}$. Then $E$ satisfies (B4) if and only if for all $e, f, g \in E$

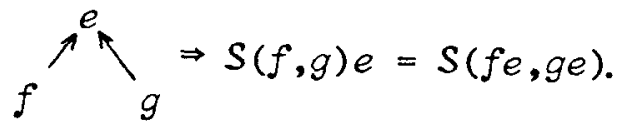

$E$ satisfies (B4)* if and only if for all $e, f, g \in E$

$\left(\mathrm{B} 4^{\prime}\right)^{*}$

$$
f^{e} \chi_{g} \Rightarrow e S(f, g)=S(e f, e g) \text {. }
$$

In the next section we use (B4') and its dual rather than (B4) and its dual.

Lemma 1. (Part of Proposition 2.3 of [2]). Let $E$ be a biordered set and $e, f$, $g \in E$. Then

$$
e \rightarrow f \rightarrow g \Rightarrow(e f) g=e(f g) .
$$

LeMma 2. Let $E$ be a biordered set and $e, f, g \in E$. Then

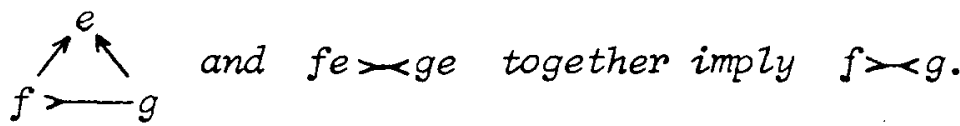

ProOF.

$$
\begin{aligned}
g f= & (g f) g & & \left(\text { since } g f>g \text { by }(\mathrm{B} 21)^{*}\right) \\
& =[(g f) e] g & & (\text { by }(\mathrm{B} 31), \text { since } g f \rightarrow g \rightarrow e) \\
& =[(g e)(f e)] g & & (\text { by }(\mathrm{B} 32)) \\
& =(g e) g & & (\text { since } g e>f e) \\
& =g & & (\text { since } g e \leftrightarrow g \text { by }(\mathrm{B} 21)) .
\end{aligned}
$$




\section{The construction and the main result}

Let $E$ be a biordered set. Observe that $><$ and $\leftrightarrow$ are equivalence relations on $E$, which we call $\mathcal{L}$ and $R$ respectively, in accordance with the usage of [2]. Let

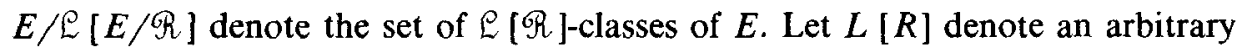
member of $E / \mathcal{L}[E / \Re]$, and $L_{e}\left[R_{e}\right]$ the $\mathcal{L}[R]$-class containing $e \in E$.

Denote the full transformation semigroup on a set $X$ by $\mathscr{T}(X)$, and the dual transformation semigroup by $\mathscr{T}^{*}(X)$. If $\alpha \in \mathcal{T}(X)$, then $\alpha^{*}$ denotes the corresponding element of $\mathscr{T}^{*}(X)$. Suppose $\infty \notin E / \mathcal{L} \cup E / \Re$. Put $A=E / \mathcal{L} \cup\{\infty\}$ and $B=E / \Re \cup\{\infty\}$.

Define

(i) $\rho: E \rightarrow \mathscr{T}(A)$ where

$$
\begin{aligned}
\rho_{e}: L & \mapsto \begin{cases}L_{x e} & \text { if } x \rightarrow e \text { for some } x \in L, \\
\infty & \text { otherwise, }\end{cases} \\
\infty & \mapsto \infty .
\end{aligned}
$$

(ii) $\lambda: E \rightarrow \mathscr{T}(B)$ where

$$
\begin{aligned}
\lambda_{e}: R & \mapsto \begin{cases}R_{e x} & \text { if } x>e \text { for some } x \in R, \\
\infty & \text { otherwise, }\end{cases} \\
\infty & \mapsto \infty .
\end{aligned}
$$

(iii) $\phi: E \rightarrow \mathcal{T}(A) \times \mathcal{T}^{*}(B)$ where

$$
e \mapsto \phi_{e}=\left(\rho_{e}, \lambda_{e}^{*}\right) \text {. }
$$

Note that $\rho$ and $\lambda$ are well-defined by (B22) and (B22)*.

REMARK. $\rho$ is the analogue of a semigroup representation. Let $S$ be a semigroup, $\mathcal{L}$ and $R$ the usual Green's relations on $S$. Denote the set of regular E-classes of $S$ by $X$. define $\bar{\rho}: S \rightarrow \mathcal{J}(X \cup\{\infty\})$ by

$$
\begin{aligned}
\bar{\rho}_{s}: L & \mapsto \begin{cases}L_{x s} & \text { if } x \Re x s \text { for some } x \in L, \\
\infty & \text { otherwise, }\end{cases} \\
\infty & \mapsto \infty .
\end{aligned}
$$

Then it is straightforward to prove $\bar{\rho}$ is a representation of $S$ (see [1]). Dually $\lambda$ is the analogue of a semigroup anti-representation.

By the following lemma, $\rho$ and $\lambda^{*}$ are morphisms of $E$ into $E(T(A))$ and $E\left(\mathscr{G}^{*}(B)\right)$ respectively.

Lemma 3. Let $E$ be a biordered set and $e, f \in E$. Then

$$
(e, f) \in D_{E} \Rightarrow \rho_{e f}=\rho_{e} \rho_{f} \text { and } \lambda_{e f}^{*}=\lambda_{e}^{*} \lambda_{f}^{*} \text {. }
$$


Proof. Suppose $(e, f) \in D_{E}$. The argument falls into two cases, each with two subcases.

(i) Suppose $e \rightarrow f$. We first show $\rho_{e}\left(=\rho_{f e}\right)=\rho_{f} \rho_{e}$. Let $L \in E / L$.

Suppose $L \rho_{e} \neq \infty$, so $x \rightarrow e$ for some $x \in L$. Then $L \rho_{e}=L_{x e}$ and $L \rho_{f} \rho_{e}=$ $L_{(x f) e}$, since by transitivity of $\rightarrow$ and by (B21) we have

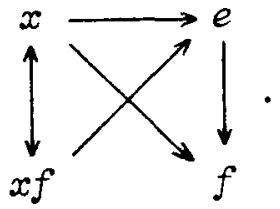

But $(x f) e=x e$ by $(\mathrm{B} 31)$, so $L \rho_{e}=L \rho_{f} \rho_{e}$.

Suppose $L \rho_{f} \rho_{e} \neq \infty$, so $x \rightarrow f$ for some $x \in L$ and $y \rightarrow e$ for some $y \in L_{x f}$, so that by (B21) and transitivity of the arrows we have

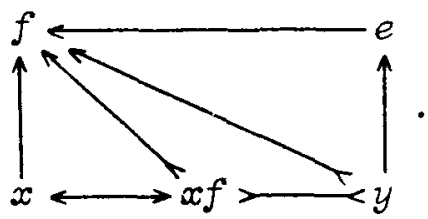

In particular $y \rightarrow f \leftarrow x$ and $y=y f \leftrightarrow x f$, so by (B4') and (B21) we have, for some $y^{\prime} \in E$

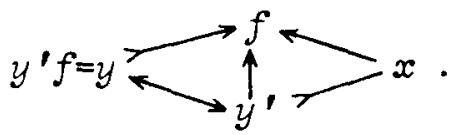

Since $y^{\prime} f \succ x f$, we have $y^{\prime} \prec x$, by Lemma 2. But

$$
y^{\prime} \leftrightarrow y^{\prime} f=y \rightarrow e,
$$

so $y^{\prime} \rightarrow e$, and so $L \rho_{e}=L y^{\prime} e \neq \infty$. Hence if $L \rho_{e}=\infty$ then $L \rho_{f} \rho_{e}=\infty$, and hence $\rho_{e}=\rho_{f} \rho_{e}$.

Now we show $\rho_{e f}=\rho_{e} \rho_{f}$. Suppose $L \rho_{e f} \neq \infty$, so $x \rightarrow e f$ for some $x \in L$. Then

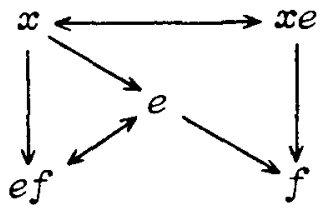


so

$$
\begin{aligned}
L \rho_{e} \rho_{f} & =L_{x e} \rho_{f}=L_{(x e) f}=L_{x(e f)} \quad \text { (by Lemma 1) } \\
& =L \rho_{e f} .
\end{aligned}
$$

Suppose $L \rho_{e} \rho_{f} \neq \infty$, so $x \rightarrow e$ for some $x \in L$, and so

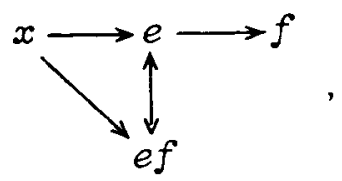

so that $L \rho_{e f} \neq \infty$. Hence $\rho_{e f}=\rho_{e} \rho_{f}$.

(ii) Suppose $e>f$. We first show $\rho_{e}\left(=\rho_{e f}\right)=\rho_{e} \rho_{f}$. Suppose $L \rho_{e} \neq \infty$, so $x \rightarrow e$ for some $x \in L$. Hence

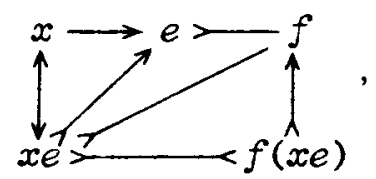

so $L \rho_{e} \rho_{f}=L_{x e} \rho_{f}=L_{f(x e)}=L_{x e}=L \rho_{e}$.

If $L \rho_{e} \rho_{f} \neq \infty$ then $L \rho_{e} \neq \infty$. Hence $\rho_{e}=\rho_{e} \rho_{f}$.

Now we show $\rho_{f e}=\rho_{f} \rho_{e}$. Suppose $L \rho_{f e} \neq \infty$, so $x \rightarrow f e$ for some $x \in L$. Then

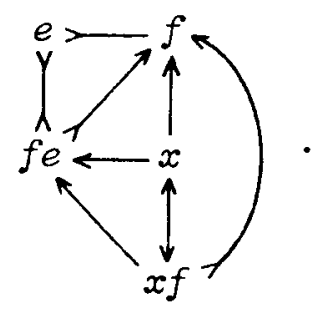

In particular $x f>f \leftarrow e$ and $x f=f(x f) \rightarrow f e$, so by $\left(\mathrm{B} 4^{\prime}\right)^{*}$ and (B21)* we have for some $x^{\prime} \in E$

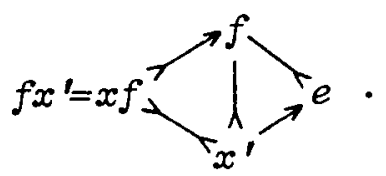


Hence

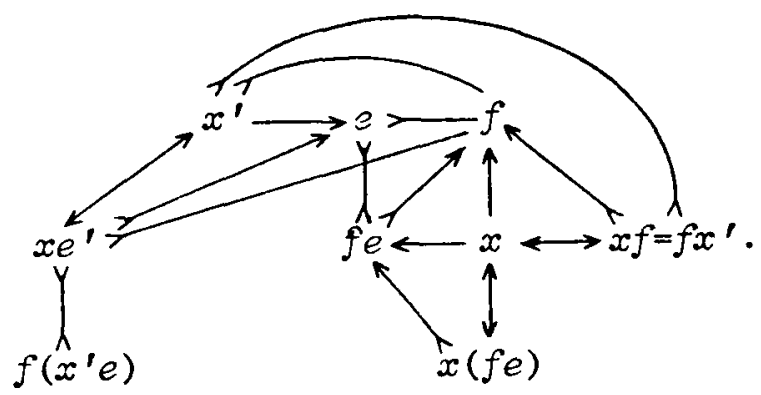

But

$$
\begin{aligned}
f\left(x^{\prime} e\right) & =\left(f x^{\prime}\right)(f e) \quad\left(\text { by }(\mathrm{B} 32)^{*}\right) \\
& =(x f)(f e) \\
& =x(f e) \quad(\text { by }(\mathrm{B} 31))
\end{aligned}
$$

so $L \rho_{f} \rho_{e}=L_{x f} \rho_{e}=L_{x^{\prime} e}=L_{f\left(x^{\prime} e\right)}=L_{x(f e)}=L \rho_{f e}$.

Suppose $L \rho_{f} \rho_{e} \neq \infty$, so $x \rightarrow f$ for some $x \in L$ and $y \rightarrow e$ for some $y \in L_{x f}$. Hence

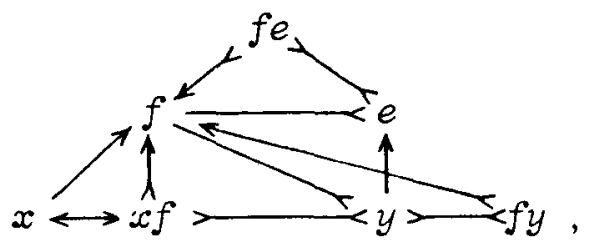

so in particular

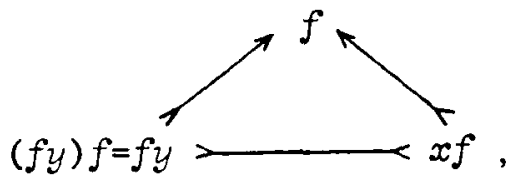

so by $\left(\mathrm{B} 4^{\prime}\right),(\mathrm{B} 21)$ and Lemma 2 we have for some $y^{\prime} \in E$

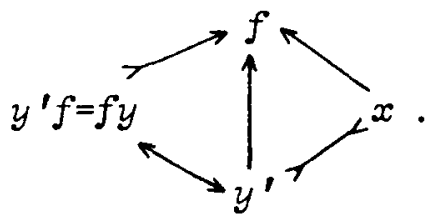


By $(\mathrm{B} 22)^{*} f y \rightarrow f e$, so

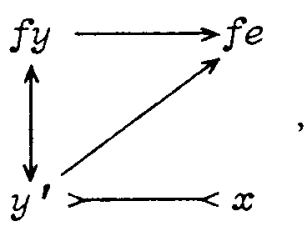

and so $L \rho_{f e} \neq \infty$. Hence $\rho_{f e}=\rho_{f} \rho_{e}$.

Thus we have shown $(e, f) \in D_{E} \Rightarrow \rho_{e f}=\rho_{e} \rho_{f}$. By the dual argument we also have $(e, f) \in D_{E} \Rightarrow \lambda_{e f}=\lambda_{f} \lambda_{e}$, that is $\lambda_{e f}^{*}=\lambda_{e}^{*} \lambda_{f}^{*}$.

LEMMA 4. Let $E$ be a biordered set and $e, f \in E$. Then

$$
\phi_{e} \phi_{f}=\phi_{e} \Rightarrow e-f \text { and } \phi_{f} \phi_{e}=\phi_{e} \Rightarrow e \rightarrow f \text {. }
$$

Proof. Suppose $\phi_{e} \phi_{f}=\phi_{e}$, so $\rho_{e} \rho_{f}=\rho_{e}$. Then $L_{e}=L_{e} \rho_{e}=L_{e} \rho_{e} \rho_{f}=L_{e} \rho_{f}$, so $x \rightarrow f$ for some $x \in L_{e}$, and $L_{x f}=L_{e}$. Hence

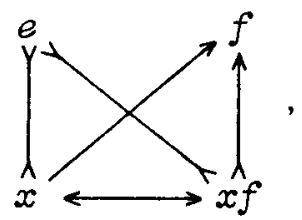

so $e>f$.

If $\phi_{f} \phi_{e}=\phi_{e}$ then $\lambda_{f}^{*} \lambda_{e}^{*}=\lambda_{e}^{*}$, so $\lambda_{e} \lambda_{f}=\lambda_{e}$, and by dual reasoning we have $e \rightarrow f$.

THEOREM 2. $\phi$ is an injective morphism from $E$ into $E\left(\mathcal{T}(A) \times \mathcal{T}^{*}(B)\right)$. $E \phi$ is a biordered subset of $E\left(\mathcal{T}(A) \times \mathcal{T}^{*}(B)\right)$ and $E \cong E \phi$ as biordered sets.

Proof. By Lemma $3, \phi$ is a morphism from $E$ into $E\left(\mathcal{T}(A) \times \mathcal{T}^{*}(B)\right)$. If $\phi_{e}=\phi_{f}$ then by Lemma 4,e $><f$ and $e \leftrightarrow f$, so $e=f$. Hence $\phi$ is injective.

We show $E \phi$ is a partial subalgebra of $E\left(\mathscr{T}(A) \times \mathscr{T}^{*}(B)\right)$.

Let $\phi_{e}, \phi_{f} \in E \phi$. If $\phi_{e}>\phi_{f}$ then, by Lemma 4, $e>f$, so by Lemma 3, $\phi_{f} \phi_{e}=\phi_{f e} \in E \phi$. Similarly if $\phi_{e} \rightarrow \phi_{f}$ then $\phi_{e} \phi_{f} \in E \phi$. Thus $E \phi$ is a partial subalgebra.

Let $\phi^{-1}: E \phi \rightarrow E$ denote the map $\phi_{e} \mapsto e$. If $\left(\phi_{e}, \phi_{f}\right) \in D_{E \phi}$ then by Lemma 4, $(e, f) \in D_{E}$, so by Lemma $3,\left(\phi_{e} \phi_{f}\right) \phi^{-1}=\left(\phi_{e f}\right) \phi^{-1}=e f=\left(\phi_{e}\right) \phi^{-1}\left(\phi_{f}\right) \phi^{-1}$, so $\phi^{-1}$ 
is a morphism of partial algebras, that is $\phi$ is an isomorphism of partial algebras. Hence $E \phi \cong E$ as partial algebras, so $E \phi$ is a biordered set, and hence a biordered subset of $E\left(\mathcal{T}(A) \times \mathcal{T}^{*}(B)\right)$.

Thus we have the following characterization of biordered sets.

COROLlaRY 1. Biordered sets are biordered subsets of the biordered sets of semigroups.

The author does not know whether a stronger result holds, namely whether biordered sets are precisely biordered sets of semigroups. Consider the free semigroup on the elements of a given biordered set, factored out by the relations of the biordered set; is the biordered set of this semigroup isomorphic to the original biordered set? If the answer is yes then the stronger result will be true, and for any given biordered set we will have produced the freest semigroup with that biordered set.

Let $E$ be a biordered set and $F$ the free semigroup on elements of $E$. Let $\rho=\left\{(z, x y):(x, y) \in D_{E}\right.$ and $\left.x y=z\right\}$, and denote the smallest congruence on $F$ containing $\rho$ by $\rho^{\#}$. To show $E \cong E\left(F / \rho^{\#}\right)$ is would be sufficient to show three things:

(i) $e, f \in E$ and $e \rho^{\#} f \Rightarrow e=f$ (no collapse);

(ii) $e \rightarrow f \Leftrightarrow e \rho^{\#} f e$, and $e>f \Leftrightarrow e \rho^{\#} e f$ (no new arrows);

(iii) $w \in F$ and $w^{2} \rho^{\#} w \Rightarrow w \rho^{\#} e \exists e \in E$ (no new idempotents).

Corollary 1 gives us

COROLLARY 2. With notation as in the preceding paragraph, conditions (i) and (ii) hold.

Proof. By Corollary $1, E$ is a biordered subset of $E(S)$, for some semigroup $S$, where we can suppose $S=\langle E\rangle$, so $S \cong F / \sigma$ for some congruence $\sigma$. If $(x, y) \in$ $D_{E}$ and $x y=z$, then $z \sigma x y$ in $F$, so $\sigma \supseteq \rho$. Thus $\sigma \supseteq \rho^{\#}$. Hence if $e, f \in E$ with $e \rho^{\#} f$ then $e \sigma f$, in $F$, so $e=f$, which proves (i). If $e \rho^{\#} f e$ then $e \sigma f e$, in $F$, so $e=f e$, and so $e \rightarrow f$. Likewise if $e \rho^{\#} e f$ then $e>-f$, which proves (ii).

The question remains whether (iii) holds. In other words, are no new idempotents created upon taking the free semigroup on a biordered set factored out by the relations of the biordered set? If (iii) holds then $E$ will be the biordered set of $F / \rho^{\#}$, and $F / \rho^{\#}$ will coincide with the free construction $E^{*}$ of Pastijn [3].

If $e_{1}, \ldots, e_{n} \in E$ and the product $e_{1} \cdots e_{n}$ is defined in $E$ with a given bracketing, then, since (i) above is true, any other bracketing yielding a defined 
product gives the same product. The proof of this relies on Theorem 2, whose proof uses the fact that $E$ satisfies (B4') and its dual, or equivalently (B4) and its dual. Without requiring $\left(\mathrm{B}^{\prime}\right)$ or its dual, it is straightforward to show that if $\left(e_{1} e_{2}\right) e_{3}$ and $e_{1}\left(e_{2} e_{3}\right)$ are defined products in $E$ then $\left(e_{1} e_{2}\right) e_{3}=e_{1}\left(e_{2} e_{3}\right)$. Note that since $E$ is a partial algebra this does not imply associative products where $n>3$. The author has looked at some special cases with $n>3$ and, with some difficulty, shown associativity in these cases without relying on (B4') or its dual. A question then is whether associativity can be shown for any $n$ in a partial algebra $E$ satisfying only (B1), (B21), (B22), (B31), (B32) and their duals. If not, precisely what role do $\left(\mathrm{B}^{\prime}\right)$ and $\left(\mathrm{B}^{\prime}\right)^{*}$ play?

\section{Acknowledgement}

The question of characterizing biordered sets was first posed to the author by his supervisor, Professor G. B. Preston.

The author gratefully acknowledges the support of a Monash Graduate Scholarship whilst undertaking this research.

\section{References}

[1] D. Easdown and T. E. Hall, 'Reconstructing some idempotent-generated semigroups from their biordered sets', Semigroup Forum, to appear.

[2] K. S. S. Nambooripad, 'Structure of regular semigroups I', Mem. Amer. Math. Soc. 22 (1979), no. 224 .

[3] F. Pastijn, 'The Biorder on the partial groupoid of idempotents of a semigroup', J. Algebra 65 (1980), 147-187.

Department of Mathematics

Monash University

Clayton, Victoria

Australia 3168 